\title{
CATALOGUING OF STABLE COMPARISONS IN THE BULGARIAN LANGUAGE BASED ON THE BULGARIAN NATIONAL CORPUS
}

\section{Palmira legurska, Teodora Krasteva}

INSTITUTE FOR BULGARIAN LANGUAGE, BULGARIAN ACADEMY OF SCIENCES

palmiralegurska@abv.bg,tera_kr@abv.bg

The article describes an attempt at cataloguing stable and unstable comparisons on the basis of material from the Bulgarian National Corpus. The main points of the work done are presented: organization of the catalogue by sections; description of the comparative words for which an explication is proposed and of the properties on which the comparison is based. The authors suggest a number of applications of the catalogue for linguistic and linguocultural purposes. A particular emphasis is put on the possibilities for integrating the catalogue into a comparative study.

Keywords: linguocultural studies, stable comparisons, patterns, catalogues, Bulgarian language

\section{REFERENCES}

Balgarski natsionalen korpus $<$ http://search.dcl.bas.bg/>

Kasabov I. 2013: Kasabov, I. Balgarska lekskikologiya i frazeologiya. T. 3. Problemi na obshtata leksikologiya. Sofia, Akademichno izdatelstvo "Prof. Marin Drinov".

Krasteva, 2017a: Krasteva, T. Ustoychivite sravneniya kato obekt na lingvokulturologiyata. - Balgarski ezik (64), 1, 87-94. <http://www.balgarski ezik.eu/12017/T-KRASTEVA-87-94-full.pdf>.

Krasteva, 2017b: Krasteva, T. Ustoychivite sravneniya kato obekt na lingvokulturologiyata. - Balgarski ezik (64), 2017,1, 87-94.

Krasteva, 2017 b: Krasteva, T. Ustoychivite sravneniya s modul na sravnenieto tsvyat v balgarskiya i ruskiya ezik. - In: Nadmoshtie i prisposobyavane. Sbornik dokladi ot Mezhdunarodnata nauchna konferentsiya na Fakulteta po slavyanski filologii, 24-25 april 2017 g. T. 2. Ezikovedski cheteniya. Kr. Aleksova (ed.), Sofia: Sofiyski universitet „Sv. Kliment Ohridski“, s. 459-464. <http://di gilib.nalis.bg/dspviewerb/srv/viewer/f97f91c8-11be-4a5c-a4ff-57822418ccdc? tk=X-RyBG-Slyk_1eCJBjM3AAAAABbRdvi.wLh5m0Od_9013kFERTiO6Q>

Kyuvlieva-Mishaykova 1986: Kyuvlieva-Mishaykova, V. Ustoychivite sravneniya v balgarskiya ezik. Sofia: Izd. na BAN.

Legurska 1982: Legurska, P. Vtorichnye leksicheskie nominatsii konkretnykh imen sushhestvitel'nykh v russkom i bolgarskom iazykakh. Sofia, Sofiiski universitet „Sv. Kliment Ohridski“, 289 s. Disertatsiya za nauchnata stepen „kandidat na filologicheskite nauki“. 
Legurska 1983: Legurska, P. Ustojchivye sravneniia s komparativnym komponentom - nazvaniem zhivotnogo v russkom i bolgarskom iazykakh. - Bolgarskaia rusistika, 1983, N 3, 46-52.

Legurska 1987: Legurska, $P$. Ustoychivi sravneniya v ruskiya i balgarskiya ezik. In: Ezikovi problemi na prevoda (ruski ezik). Sofia, Nauka i izkustvo, s. 135141.

Legurska 2003: Legurska, P. Fragmenti ot ezikovata kartina prez prizmata na vtorichnoto nazovavane (osnovi na nauchnoizsledovatelski proekt). - Chuzhdoezikovo obuchenie, 5, 5-22. <www. belb.net>

Legurska 2006: Legurska, $P$. Fragmenti ot ezikovata kartina prez prizmata na vtorichnoto nazovavane (varhu material ot balgarski, ruski, cheshki, srabski, frenski, angliyski ezik) - st.n.s. d-r Palmira Legurska - rakovoditel, st.n.s. d-r Nichka Becheva, dots. d-r Dimitar Veselinov, n.s. d-r Tsvetana Avramova, n.s. d-r Maria Lilova. Sadarzhatelna i tehnicheska unifikatsia: Ivo Panchev, st.n.s. d-r Palmira Legurska (Danni po NATSID, Sofia, $155 \mathrm{~s} .<$ http://georgesg.info/belb/ projects/proektpm/4.\%20> Onomas.katalog-prehodi(Ru-Bg).pdf, data na dostap: 11.07.2018)

Legurska 2009: Legurska, P. Sapostavitelno-tipologichen analiz na vtorichnata nominatsiya na predmetnite imena v balgarski, ruski, srabski, cheshki, frenski i angliyski ezik. Teoretichna osnova na proekta. 2009. Rakopis, depoziran v NATSID, chast I. Inv. № ND 51/2009.

Legurska 2011: Legurska, P. Sapostavitelni leksikalni analizi i osnova za sapostavka. Sofia, Izdatelstvo „Eto“, 226 str.

Legurska et al. 2009: Legurska, P., N. Becheva, Tsv. Avramova, D. Veselinov, M. Lilova, I. Panchev. Sapostavitelno-tipologichen analiz na leksikata v rodstveni i nerodstveni ezitsi (varhu material ot balgarski, ruski, srabski, cheshki, frenski i angliyski ezik). II chast. Depoziran rakopis v NATSID, UDK: 801. Signatura ND II 18791, № ND 65/2009, Sofia, 1093 str.

Legurska, Panchev 2014: Legurska, P, I. Panchev. Obrazni metafori (onomasiologichen katalog). Elektronno spisanie Dzyalo, br. 3. <http://abcdar.com/magazine_ I.php ISSN 1314-9067>.

Legurska 2015: Legurska, P. Sapostavitelni analizi i natsionalna spetsifika na leksikata. Sofia, Izdatelstvo „Eto“.

Rechnik na balgarskia ezik (online). http://ibl.bas.bg/rbe/.

Skorupka 1958: Skorupka, St. Idiomatizmy frazeologiezne w jezyku polskim i ich geneza. - In: Slavyanskaia filologiia. Москва. Pilz 1980: Pilz, K. D. Phraseologie. Stuttgart.

Yanev 2013: Yanev, B. Obraznite sravneniya s antropotsentrichen harakter v balgarskiya $\mathrm{i}$ angliyskiya ezik. Izsledvane i rechnik. Plovdiv. Universitetsko izdatelstvo „Paisiy Hilendarski“.

Assoc. Prof. Palmira Legurska, PhD; Teodora Krasteva Department of Ethnolinguistics Institute for Bulgarian Language, Bulgarian Academy of Sciences 52 Shipchenski prohod blvd., bl. 17, 1113 Sofia, Bulgaria 